\title{
Polyarteritis Nodosa: A Rare Form of Presentation
}

\author{
Sara Camões*1, María Rabadán López ${ }^{2}$ and Marta Fernández Morales ${ }^{3}$ \\ ${ }^{1}$ Internal Medicine Service, Hospital Pedro Hispano, Matosinhos, Portugal \\ ${ }^{2}$ Internal Medicine Service, Complejo Hospitalario Universitario de Badajoz, Spain \\ ${ }^{3}$ Internal Medicine Service, Hospital do Salnés, Pontevedra, Spain
}

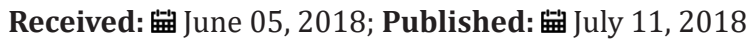

*Corresponding author: Sara Camões, Hospital Pedro Hispano, 4464-513 Senhora da Hora, Matosinhos, Portugal

\begin{abstract}
Poly arteritis nodosa (PAN) is a necrotizing vasculitis of medium caliber arteries in which the involvement of small vessels is not typical.The most affected organs are skin, gastrointestinal tract and peripheral nerves. In our case report, PAN presented with pain, swelling and altered sensation of the left leg, mimicking deep vein thrombosis, soft tissue infection or even a compartment syndrome. We present this case to raise awareness to this rare but possible form of PAN presentation, that will need a distinct approach.
\end{abstract}

Keywords: Polyarteritis Nodosa; Systemic Vasculitis; Skin; Connective Tissue Diseases

Abbrevations: PAN: Polyarteritis Nodosa; ANCA: Antineutrophil Cytoplasmic Antibodies; FFS: Five Factor Score; CT: Computerized Tomography; MRI: Magnetic Resonance Imaging; ANA: Anti-dsDNA: Anti-Double Stranded DNA

\section{Introduction}

Polyarteritis nodosa (PAN) is a necrotizing vasculitis of medium caliber arteries in which the involvement of small vessels is not typical (that is glomerulonephritis is unusual), and is not associated with the presence of antineutrophil cytoplasmic antibodies (ANCA). It affects similarly both sexes and the most frequent age of presentation oscillates between the fifth and sixth decade of life. Although PAN is considered a primary vasculitis, there are forms associated with viral infections, mainly hepatitis B virus [1].

\section{Case report}

We present a 45-year-old Chinese man, living in Spain for fifteen years, who works as a store manager. Smoker of 10 cigars a day without other toxic habits, no known drug allergies, does not take any usual treatment and denies recent trips. He referred a ten-day history of muscular pain and swelling of the left calf muscle without erythema, local heat or fever. At the emergency department, he seemed to have a positive Homans sign and a disproportionate left lower limb pain due to the absence of inflammatory signs. D-dimer was discreetly elevated $(710 \mathrm{ng} / \mathrm{ml})$, with a C-reactive protein of $6 \mathrm{mg} / \mathrm{dL}$ and 14,000 leukocytes with neutrophilia. An ultrasound with venous Doppler ruled out deep vein thrombosis. In addition, a computerized tomography (CT) of the left leg was performed, as the patient maintained intense pain refractory to analgesic treatment; the CT showed interfascial fluid in the medial gastrocnemius, without air or intramuscular liquid collections, findings that are compatible with a case of necrotizing fasciitis in an initial stage. With this result, Infectious diseases and
Traumatology were contacted, and an urgent surgical intervention was performed. From the macroscopic point of view, a muscular ischemic territory of $3 \times 3$ centimeters was evidenced and samples for microbiological studies were taken. Treatment with imipenem and linezolid was started while waiting for the microbiological studies, that later turned out to be negative (both the blood cultures and the intraoperative samples).

Within 48 hours of hospital admission, the patient began abdominal pain, predominantly in the right hypochondrium, with a colic character and fluctuating intensity. In the following days, cholestasis and progressive elevation of acute phase reactants appeared; abdominal ultrasound showed biliary mud and a discrete edema of the gallbladder wall. At the same time, the patient developed purpuric and palpable skin lesions, mainly on the right lower extremity, which in the following days extended to the contralateral extremity and lower part of the trunk, both in the anterior and posterior regions. Likewise, muscular pain progressed to the right leg. To clarify the etiology of the pain a magnetic resonance imaging (MRI) of the lower limbs was requested (Figure 1), which revealed the existence of multiple scattered foci of myositis and fasciitis of several muscular areas, predominating in the right limb. Given the clinical course, which was not typical of necrotizing fasciitis, and all these new data, the suspicion of vasculitis was established and the Service of Systemic Autoimmune Diseases was consulted. The study was then extended: erythrocyte sedimentation rate of $72 \mathrm{~mm} /$ hour, negative antinuclear antibodies (ANA), anti-double stranded DNA (anti-dsDNA), ANCA, 
rheumatoid factor and cryoglobulins, normal complement and immunoglobulins. Viral serologies were negative for hepatitis B, C and human immunodeficiency virus.

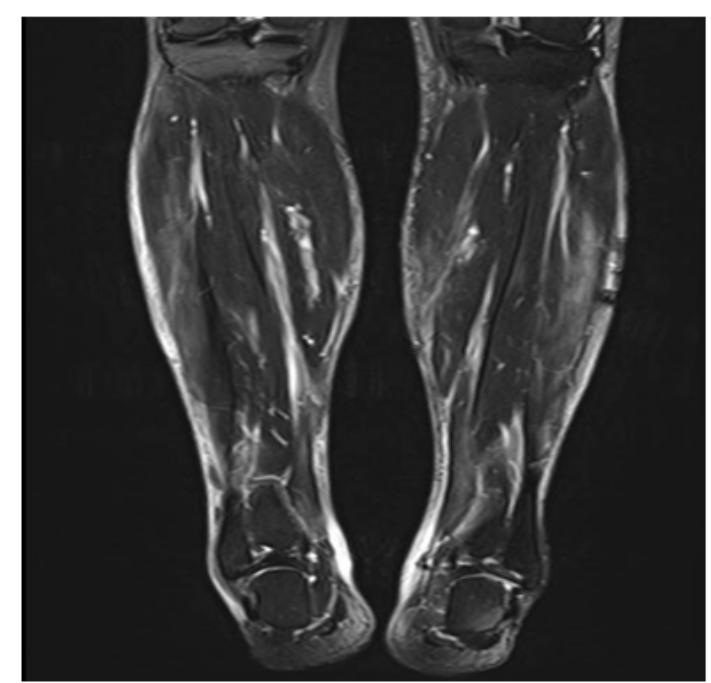

Figure 1: Magnetic resonance of lower limbs showing multiple scattered foci of myositis and fasciitis of several muscular areas, predominating in the right limb.

Electromyography showed only a mild to moderate sensory neuropathy of the left median nerve. We then performed a CT angiography that showed occlusion of the distal left tibial anterior artery, as well as thickening and parietal edema of the duodenum wall, ileum and ascending colon (Figure 2), without signs of vasculitis activity in the arteriography. Finally, the histology of the sample obtained during the surgical intervention allowed us to identify findings of medium vessels vasculitis, compatible with the diagnosis of polyarteritis nodosa (PAN). Following this diagnosis, antibiotics were withdrawn and intravenous bolus of methyl prednisolone was started at $250 \mathrm{mg}$ a day for three days, followed by prednisone $1 \mathrm{mg} / \mathrm{kg} /$ day. After performing the CT angiography and assuming the intestinal symptoms as a manifestation of vasculitis, induction treatment with cyclophosphamide was initiated. The patient evolved favorably, allowing to continue outpatient treatment with cyclophosphamide and descending corticosteroid regimen.

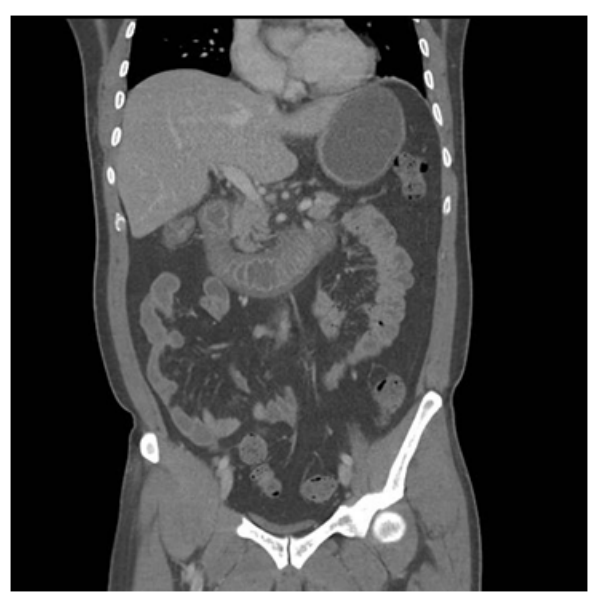

Figure 2: Abdominal CT angiography showing thickening and parietal edema of duodenum wall, ileum and ascending colon.

\section{Discussion}

According to the American College of Rheumatology classification's criteria for PAN, it is defined that at least three of the ten criteria must be present to obtain a sensitivity of $82,2 \%$ and a specificity of $86,6 \%$. The main diagnostic test still is the histology that confirms vasculitis [1]. The most affected organs are skin, gastro intestinal tract, kidney, peripheral nerves, heart, liver, pancreas and testicles. The peculiarity of this case lies in its presentation form, localized at the lower limbs, with pain and swelling that could mimic a deep vein thrombosis, soft tissue infection or even a compartment syndrome. Myalgia occur in about $51 \%$ of PAN cases, which may be due to the involvement of intramuscular arteries, the involvement of the peripheral nervous system or, more rarely, to myositis [2]. PAN should be considered as a differential diagnosis in cases in which MRI images show diffuse or unequal muscle signal changes. Muscle involvement in PAN is characterized by the presence of lesions centered on vessels, and may be accompanied by fascial or periosteal enhancement [3].

Treatment will depend on the presence of the severity criteria proposed by the French Vasculitis Study Group, known as Five Factor Score (FFS) that focus on severe gastrointestinal, renal, cardiac and central nervous system involvement. These factors have a prognostic value, so that the greater the number of factors present, the higher the mortality rate. If FFS is 0 , prednisone can be started at $1 \mathrm{mg} / \mathrm{kg} /$ day to induce remission. However, if there is a FFS $\geq 1$, cyclophosphamide $(15 \mathrm{mg} / \mathrm{kg}$ monthly) should be associated for at least 6 months until achieving remission; in these cases, prednisone can be replaced by bolus of methylprednisolone for the first 3 days of treatment. Subsequently, if FFS $=0$ prednisone should be maintained at $1 \mathrm{mg} / \mathrm{kg} /$ day for one month before initiate a descending pattern. With a FFS $\geq 1$, the corticosteroid regimen is the same, but we should associate azathioprine $(2 \mathrm{mg} / \mathrm{kg} /$ day $)$ or methotrexate (until achieving doses of 20-25 mg/weekly) as maintenance treatment, once remission with cyclophosphamide is achieved, especially when prednisone cannot be tapered below 15$20 \mathrm{mg} /$ day $[1,4]$.

\section{References}

1. Hernández-Rodríguez J, Prieto-González S, Alba MA, Forns X, Cid MC (2014) Poliarteritis nudosa. En: Cervera R. Enfermedades autoinmunes sistémicas. Diagnóstico y tratamiento $\left(5^{\text {th }}\right.$ Edn. $)$, Madrid: Editorial médica Panamericana, SA, pp. 134-145.

2. Calvo R, Negri M, Ortiz A, Roverano S, Paira S (2017) Myositis as the initial presentation of panarteritis nodosa. Reumatol Clin S1699258X(17): 30169-30169.

3. Kang Y, Hong SH, Yoo HJ, Choi JY, Park JK, etal. (2016) Muscle Involvement in Polyarteritis Nodosa: Report of Eight Cases With Characteristic Contrast Enhancement Pattern on MRI. AJR Am J Roentgenol 206(2): 378-384.

4. Hernández-Rodríguez J, Alba MA, Prieto-González S, Cid MC (2014) Diagnosis and classification of polyarteritis nodosa. J Autoimmun 48-49: 84-89. 


\section{ISSN: 2574-1241}

DOI: 10.26717/BJSTR.2018.06.001389

Sara Camões. Biomed J Sci \& Tech Res

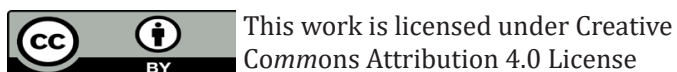

Submission Link: https://biomedres.us/submit-manuscript.php

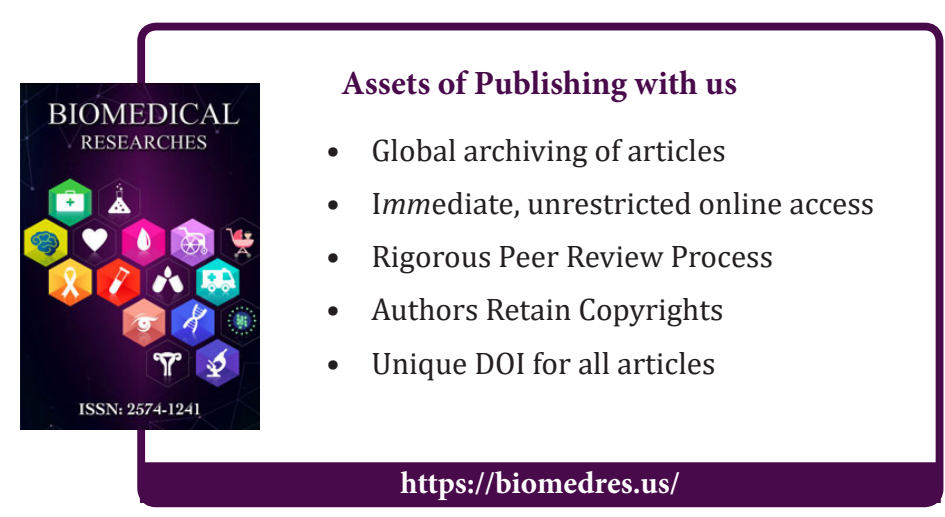

